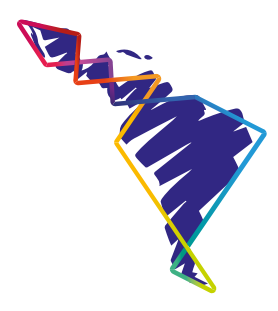

\title{
Gordofobia: existencia de un cuerpo negado. Análisis de las implicaciones subjetivas del cuerpo gordo en la sociedad moderna
}

\author{
Fat phobia: existence of a denied body. \\ Analysis of subjective implications of the \\ fat body in modern society
}

\section{Fobia gordurosa: existência de corpo negado. Análise das implicações subjetivas do corpo gordo na sociedade moderna}

\section{Gabriela Quirós Sánchez ${ }^{1}$}

\begin{abstract}
Resumen
El presente manuscrito tiene como finalidad propiciar la reflexión en torno a la construcción social del cuerpo gordo, desde el análisis de la gordofobia como un elemento de aprendizaje psicosocial que tiene como fin la categorización de la valía personal, propiciando un posicionamiento diferenciado para las personas cuyas corporalidades no están determinadas dentro de las rígidas estructuras de la estética actual. Los aprendizajes sobre el cuerpo tienen efectos diferenciados sobre la vida de las personas; la construcción de género, las enseñanzas sociales y la influencia de la legitimación familiar del discurso discriminatorio son algunos de los mecanismos por los que la gordofobia permea la autopercepción corporal e influye en la forma en la que nos tratamos e interactuamos con los demás. Aceptar la diversidad corporal en la región latinoamericana no solo tiene que ver con el reconocimiento de las formas de nuestros cuerpos, sino también, con la aprobación de la herencia histórica de nuestro mestizaje. Siendo
\end{abstract}

1 Licenciada en Género y Desarrollo. Licenciada en Psicología. Académica División de Educación Rural, Universidad Nacional de Costa Rica. ORCID: https://orcid.org/0000-0003-0350-838X . Correo electrónico: gabriela.quiros.sanchez@una.cr 
esta una reivindicación pendiente que se ve asediada por la influencia de la moda, el mercado y la mirada heteropatriarcal sobre el cuerpo.

Palabras claves: gordofobia, educación, género, discriminación.

\begin{abstract}
The present manuscript is intended to encourage reflection on the social construction of fat body, from analysis of fatphobia as an element of psychosocial learning that aims at categorization of bodies, promoting a differentiated positioning for people whose bodys are not determined within rigid structures of current body aesthetic. Learning about the body has different effects on people's lives. The gender construction, social learning and family's legitimization of discriminatory discourse is some of the mechanisms by which fat-phobia permeates bodily self-perception and influences the way we treat and interact with each other. Recognizing Latin American body diversity not only has to do with recognizing the forms of our bodies, but also has to do with recognizing and accepting the historical heritage of our blending. This is a pending claim by fashion influence, the market and the heteropatriarchal look on the body.
\end{abstract}

Keywords: Fatphobia, education, gender, discrimination.

\title{
Resumo
}

O presente artigo tem como objetivo incentivar a reflexão sobre a construção social do corpo gordo, a partir da análise da fatfobia como elemento de aprendizado psicossocial que visa à categorização dos corpos, promovendo um posicionamento diferenciado para pessoas cujos corpos não são determinados dentro do rígido estruturas da estética corporal atual. Aprender sobre o corpo tem efeitos diferentes na vida das pessoas. A construção de gênero, a aprendizagem social e a legitimação familiar do discurso discriminatório são alguns dos mecanismos pelos quais a gordura-fobia permeia a autopercepção corporal e influencia a forma como tratamos e interagimos uns com os outros. Reconhecer a diversidade corporal latinoamericana não tem apenas a ver com o reconhecimento das formas de nossos corpos, mas também tem a ver com o reconhecimento e aceitação da herança histórica de nossa fusão. Esta é uma reivindicação pendente por influência da moda, o mercado eo olhar heteropatriarcal sobre o corpo.

Palavras chaves: Fobia gordurosa, educação, gênero, discriminação.

\section{Introducción}

El cuerpo representa un espacio territorial en disputa, inmerso en variables económicas, sociales, estéticas, ideológicas, raciales, sexistas, entre otras, que interactúan entre sí, configurando un tejido social complejo de categorizaciones, aprendizajes y simbolismos determinados por la evolución sociocultural y los intereses hegemónicos del deseo heteropatriarcal. 
La gordura corporal, bajo este escenario, constituye un antivalor asociado a aquello determinado como indeseable, enfermo, asexuado y de poco valor social. Las implicaciones subjetivas del cuerpo gordo en la sociedad moderna conllevan un debate entre la percepción racional de la visión médica sobre la gordura y la idea de quienes experimentan el rechazo social por existir desde un cuerpo negado.

Bajo la lógica moderna, la mercantilización del cuerpo ha propiciado el consumo exacerbado de símbolos, sin importar las consecuencias físicas o emocionales que la demanda social por construir la "figura ideal" pueda traer a los sujetos. De modo que, bajo la lógica de la complacencia simbólica, los individuos predisponen sus corporalidades a procesos violentos que van desde la restricción alimentaria, hasta la trasformación quirúrgica, todo esto, por encajar dentro de un patrón normativo que vende la ilusión de ser "aceptado" en el nivel social.

De esta manera, se pierde de vista que el mestizaje latinoamericano ha creado una diversidad corporal compleja que evidencia la herencia ancestral de los pueblos originarios, las raíces africanas y sus matices históricos, así como las huellas de la colonización europea. De modo que la idea del físico que se mercantiliza en Occidente incide directamente dentro de los procesos subjetivos y la configuración del contexto social regional, puesto que cuestionar el cuerpo, es hacerlo con la existencia misma.

Por lo anterior, las reflexiones aquí planteadas buscan introducir la gordofobia dentro del debate académico, como parte de un esfuerzo por conceptualizar el cuerpo gordo desde otros lugares, reivindicando la existencia de las personas desde sus diversidades y focalizando la acción sentipensante en el reconocimiento de la experiencia vivida como un factor que enmarca de forma humanista el reconocimiento de la gordura corporal y sus implicaciones en el nivel social.

\section{El cuerpo gordo en la sociedad latinoamericana moderna}

Las trasformaciones económicas, sociales, políticas e ideológicas que ha traído consigo la modernidad a la región, han trasformado radicalmente la forma en la que los individuos se reconocen entre sí y, sobre todo, las condiciones bajo las cuales se adscriben valor en el nivel simbólico y de facto. Para esto, las diversas instituciones sociales han procurado por la segmentación poblacional a través de la categorización y la diferenciación de las personas por estratos, siendo algunas de estas sobre valoradas por sus condiciones, financieras, educativas, simbólicas, entre otros. 
Uno de los elementos sociales que ha emergido con gran fuerza a partir de los avances médicos y tecnológicos está relacionado con la estética corporal. La concepción del cuerpo desde parámetros físicos posiciona a los sujetos de forma diferenciada dentro de las sociedades, donde los simbolismos asociados al cuerpo potencian la aceptación y la admiración social.

La belleza corporal es un concepto que debe ser entendido desde la lógica capitalista como un importante símbolo que "hace vender". Por lo que la industria (sobre todo de la moda y la perfección) ha necesitado "liberar y emancipar", en una palabra "redefinir" el cuerpo (Baudrillard, s. f. citado en Martín, 2002). Las trasformaciones físicas son el resultado de un proceso de asimilación de la demanda social por la construcción de figuras estilizadas, de modo que la lógica en la "venta corporal" está relacionada no solo a parámetros heteronormativos, sino, además, a estructuras sexistas y discriminatorias que relegan a las corporalidades disidentes a espacios de ocultamiento y conmiseración.

Sin embargo, la gordofobia representa un fenómeno social naturalizado dentro de la mayoría de las sociedades occidentales que ha sido asumido como una forma de interacción "normal" a partir de la patologización de todo tipo de gordura en el cuerpo. Dado que el cuerpo gordo ha sido simbolizado como enfermo, cuyas significaciones están asociadas con la burla, el aislamiento social, la asexualidad y la muerte prematura.

Al ser las mujeres quienes experimentan en mayor medida y con un parámetro superior de rigidez, la censura de la grasa en el cuerpo, con relación al de los hombres, dentro de las interacciones sociales, explica la relación entre la obesidad y la depresión, y las formas en que una puede ocasionar e influir sobre la otra, pues existe una conexión significante entre las mujeres con un alto índice de masa corporal (IMC) y pensamientos de suicidio (Díaz y Díaz, 2008).

El argumento de la gordura corporal recae en la unidireccionalidad que se ha construido con relación al tema, al haberse estructurado una asociación simbólica entre el cuerpo gordo y la visión más negativa de este, sin que medie reflexión, ni quiebre epistémico entre la noción de cada concepto. La perspectiva en torno a la obesidad se presenta como uno de los grandes problemas de salud que azotan a los principales países industrializados. Aunque, esta es una aproximación parcial cuyos referentes están enfocados en la visión médica, de salud pública o desde el problema social (Magallares, Morales y Rubio, 2010).

El poder adscrito a la noción médica ha impedido el reconocimiento de la gordura corporal desde otros lugares, de modo que los discursos asociados a la grasa, se 
encuentren cargados de nociones prejuzgadas que aluden de manera indiscutible, a estados paupérrimos de salud física o emocional, a un deterioro paulatino de las habilidades sociales o personales en torno al desempeño académico o profesional, así como una sistemática desvalorización del sujeto como individuo, de modo que las personas gordas, dejan de ser reconocidas como tales y pasan a configurar grupos carentes de poder, de significados y por ende constituyen cuerpos negados que son relegados a espacios, labores y funciones predeterminadas y con bajo reconocimiento social y económico.

En el 2016, investigando sobre el tema con estudiantes de la Universidad Nacional de Costa Rica, enfaticé la necesidad de reconocer si las personas que estaba encuestando se sentían gordas; recuerdo que estando en la Escuela de Ciencias del Movimiento Humano, encuesté a una estudiante de la carrera de Educación Física, cuyas formas corporales encajaban dentro del estereotipo erótico actual asociado a la feminidad. Esta estudiante, mientras avanzaba al responder el cuestionario, leía en voz alta cada una de las preguntas ahí planteadas. Al dar vuelta a la página y leer más bajo: ¿Te sentís gorda/o?, exclamó con fuerza, ¡Uff, gordísima!, al interrogar por esta reacción, empezó a enumerar cada una de las características de su cuerpo que la hacían sentirse así, como si sus anchas caderas o sus piernas gruesas, producto de su herencia afrocostarrisence, determinaran su calidad académica, su valor personal o su futuro profesional.

Sentirse una persona gorda configura un estado mental propiciado desde la construcción de patrones culturales que sobrevaloran la delgadez corporal y que la legitiman dentro de las diversas interacciones sociales, de modo que la adherencia a los aprendizajes relacionados con el peso, el color, el tamaños y las formas correctas, aceptadas y validadas en una sociedad repercuten de forma directa o indirecta en la relación establecida entre los sujetos, sus esquemas mentales y el cuerpo real en el que se habita.

Sin embargo, tenemos una responsabilidad latente sobre la herencia de patrones conductuales que privilegian a los cuerpos delgados por sobre las corporalidades gordas. El desarrollo de estados de disconformidad se traduce en sentimientos de vergüenza, culpa, desvalía, conmiseración, entre otros, están nutridos por una amplia gama de mensajes directos o indirectos que censuran la gordura y cuya fuente representa gran importancia para el sujeto. Hablamos de mensajes violentos disfrazados de recomendaciones "bien intencionadas" que son emitidos de forma indiscriminada por las figuras primarias, familiares cercanos, pares o incluso desconocidos. El acto de prejuzgar el cuerpo ajeno es más común de lo que parece. 
La teoría del aprendizaje plantea que el individuo va adquiriendo una conducta desde su niñez, por medio de observación e imitación; estas observaciones comienzan en el entorno más cercano que es el de la familia y van externalizando a otros contextos como lo es la escuela y los grupos pares (Darling y Cumsille, 2003).

De este modo, establecemos una relación direccional entre los aprendizajes derivados de los mensajes de aceptación o rechazo corporal emitidos por el sistema social, las figuras primarias y el círculo cercano, con relación al cuerpo propio y al ajeno. Estos mensajes se dan de forma consciente o inconsciente a través de las construcciones simbólicas de la gordura en sociedad, el lenguaje verbal y no verbal, se expresa, además, a través de silencios, patrones conductuales, relaciones sociales, chistes, entre otros.

En términos de socialización, las personas aprendemos patrones conductuales que determinada nuestro lugar dentro de los colectivos. En la adolescencia, por ejemplo, el posicionamiento social es trascendental para la construcción del yo, pues configura el espacio de separación de las figuras primarias para la edificación de los esbozos de quien se aspira a ser.

Los mensajes tácitos o directos relacionados con el cuerpo impactan de forma diferenciada el proceso de construcción del yo de mujeres y hombres en esta etapa, independientemente, del país en el que viva, dado que la educación sexo-genérica les posiciona de manera desigual de cara a las exigencias sociales demandadas sobre el cuerpo para unas y otros dentro de nuestros países.

Sin embargo, en la vida adulta, la relación con el cuerpo no dista mucho de las experiencias adolescentes. Múltiples son los ejemplos de casos de acoso laboral que incluyen de forma explícita comentarios despectivos asociados a las habilidades profesionales de los sujetos medidos desde parámetros gordofóbicos; y es que pareciera que llamar gorda a una persona constituyera una ofensa planificada con el objetivo de lastimar.

Estamos tan habituados a reconocer que la gordura corporal está mal, que se nos complica aceptarnos gordos, o determinarlo en el otro, de modo que empleamos diminutivos que buscan suavizar un hecho ineludible: el cuerpo gordo es negado, pues admitir su existencia propicia la tolerancia de una diversidad física que no es atractiva a la mirada heteropatriarcal, que no aporta significativamente al sistema económico, pues se parte de la noción del organismo enfermo que necesita ser atendido y que por ende, no es suficientemente capaz de cumplir con las exigencias productivas actuales y además, que no tiene un espacio positivo dentro de las representaciones sociales, pues la gordura, en todas sus formas, se encuentra 
excluida y censurada dentro del imaginario sociocultural, a menos que se busque su descalificación o censura.

Alemany (1993, citado en Martínez, 2004), refiere que el imaginario en la construcción de la noción del cuerpo ideal tiene una importación diferente para las mujeres en comparación con los hombres, sin que esto sea una constante universal, dado el bombardeo de signos a los que la corporalidad se ve enfrentada en la actualidad. Sin embargo, en el nivel social se muestra una tendencia que otorga mayor atención e importancia hacia la forma física de las mujeres, ya que el calificativo "gorda" es percibido en muchos casos como insulto, frente a la palabra "gordo", que es asumida como una característica personal.

La gordura en el cuerpo de las mujeres es totalmente distinta a la experiencia en el cuerpo de varones, incluyendo también a las maricas. Ejemplifico desde mi contexto geopolítico, la descripción de cómo debe ser un chico gordo promedio en chile: el gordito buena onda, el gordito parrillero, bueno para la cerveza, el osito, el fuerte o matón, el gordo tatuado, el gordo peludo, el gordo heterosexual protector, de igual forma deseable sexualmente frente a una chica heterosexual. Las chicas gordas: la eterna amiga de la chica bonita, inteligente y algo nerd, como un libro con patas, sin cuerpo sexuado, la gordita simpática, agradable, aduladora, la gorda bonita (Álvarez, 2014, p.70).

Si bien, no podemos establecer generalidades sobre un tema tan subjetivo como la relación con el cuerpo propio, si es necesario hacer un llamado a la reflexión en términos del impacto de la conexión con nuestro ser y las proyecciones sobre el físico de los demás. Es decir, los aprendizajes asociados al cuerpo son trasformados de forma inconsciente en demandas personales que se proyectan sobre los otros, sin que medie reflexión sobre el origen de ellas o los efectos que estas puedan generar sobre el desenvolvimiento y la autoestima de los individuos que les recibe.

Como ya se estableció, llamar "gorda" a una persona, cuya experiencia de vida en relación con su cuerpo haya distorsionado su autopercepción, puede desencadenar procesos autolesivos como anorexia o bulimia; trastornos de la conducta alimentaria que se potencian a partir de la sobre exaltación de la delgadez en el nivel sociocultural.

En términos psicosociales, las implicaciones subjetivas de las proyecciones inconscientes de las demandas personales sobre los otros podrían potenciar trastornos de tipo ansioso, de estado del ánimo y por supuesto, de conducta alimentaria. Sin embargo, cabría detenerse y reflexionar sobre las motivaciones personales que llevan a un sujeto a depositar en los otros sus propias demandas corporales. Desde 
una perspectiva foucaultiana, vivimos bajo un panóptico perpetuo donde somos jueces y parte del control social sobre los cuerpos.

Si bien, existe una tendencia global por homogenizar los patrones corporales desde la construcción de una realidad donde solo importan los cuerpos delgados, principalmente desde nuestro contexto geopolítico, es necesario reflexionar sobre la intencionalidad con la que estos mensajes son emitidos, pues responden a intereses económicos y políticos de carácter dominante que son trasmitidos y legitimados a través de múltiples medios, siendo la educación formal y no formal el principal canal de difusión y herencia de los estándares de comportamiento y vinculación de los sujetos consigo mismos, su círculo primario y su entorno.

El proceso de construcción de la realidad social se hace posible en dos momentos: la institucionalización y la legitimación del comportamiento humano. El primero, se origina en la tendencia de habituación del ser humano en interrelación con sus congéneres, su medio y su contexto, logrando establecer patrones de conducta social acordes a su status social y a los roles que desempeña dentro del grupo al que pertenece. Esta habituación es de tal magnitud que los patrones institucionalizados se convierten en un elemento de control social; (...) la legitimación constituye el segundo momento de la construcción de la realidad social y tiene la tarea de convertir dichas pautas de comportamiento en «lícitas», permitidas o establecidas, las mismas que serán reproducidas y afianzadas constantemente a través del proceso de internalización y externalización (Berger y Luckmann, 1972 citado en Chegne, 2012, p. 9).

Las estructuras mentales desarrolladas a partir de los aprendizajes gordofóbicos derivados de la cultura, la familia, la medicina, etc., son tan rígidos y tienen cimientos tan fuertes sobre las percepciones de las personas, que una vez que alguien es gordo, nunca deja de serlo, aun cuando su realidad corporal cambie.

La noción de la gordura pasada constituye un latente persecutorio que arrastra el individuo en sociedad, de modo que el recuerdo de lo que fue se vuelve una sombra que es traída al presente por medio de comentarios y simbolismos, propiciados por familiares o desconocidos que posicionan al sujeto como ejemplo de éxito, de aspiración, mientras cumpla con la norma social del "cuido corporal", situación que se fundamenta bajo el disciplinamiento en términos de ejercicio físico o restricción nutricional. Sin embargo, existe un escenario alterno que es percibido de forma amenazante y se funda en la supervisión de la conducta alimentaria bajo el recuerdo de "lo que fue". 
La gordura marca el cuerpo, pero, también, las relaciones sociales establecidas entre los sujetos, de modo que dejar de ser una persona gorda, no necesariamente, transforma los vínculos entre el ser y su físico o entre los individuos, puesto que la gordura en sociedad está tan mal categorizada, que las heridas personales derivadas de las burlas, los comentarios "amistosos", los consejos nutricionales, etc., constituyen verdaderos conflictos internos, que no se superan con bajar de peso. La autopercepción se trastoca y la herida emocional se reaviva cuando se aspira a alcanzar un patrón estético diseñado para y desde un cuerpo irreal.

Parece existir un consenso generalizado en que el atractivo personal es un constructo social multidimensional. Afirma Lloyd que "no es un objeto, sino un valor y, como cualquier otro valor, requiere para su existencia de una relación extrínseca con alguna persona en cuya conciencia emerge [...] la belleza no es una cualidad innata al objeto [o a la persona], sino una propiedad percibida con respecto a ella por otra" (Lloyd, 1923, p. 225). Quiere esto decir que la belleza personal es un intangible, que no depende tanto del sujeto en que supuestamente reside, como del que juzga (Martín, 2002, p. 1).

De esta manera, es a través de las relaciones sociales donde se manifiesta el poder entre los sujetos a partir de ejercicios conscientes o inconscientes de control sobre el otro, de modo que comentarios que aluden al disciplinamiento corporal en términos de restricción alimentaria o bien bajo el precepto de consejos en torno a la intensidad o frecuencia en el entrenamiento físico, constituyen elementos emergentes que ameritan atención, sobre todo, cuando la relación entre los individuos no es cercana, o bien, cuando es justamente esto lo que potencia un abuso en la confianza que desemboca en palabras hirientes con relación al cuerpo ajeno.

\section{Conclusiones}

El cuerpo gordo ha sufrido una serie de trasformaciones históricas que le han posicionado de forma diferente en sociedad. Las significaciones asociadas a la gordura han sido cambiadas a partir de las implicaciones y de las demandas económicas de cada época. La modernidad ha traído consigo una serie de procesos que ha industrializado y mercantilizado la imagen corporal de formas tan diversas, tan sutiles y a la vez, tan directas que se han incorporado a la cotidianidad como elementos de interacción, donde la burla o el desprecio para con el cuerpo gordo, constituye una forma naturalizada de relacionamiento, cuyas consecuencias físicas y emocionales son subestimadas.

El uso de la imagen del cuerpo gordo está adscrito a una categoría indeseada, el físico no delgado, salido del molde estético dominante, establece carencias de 
representaciones sociales positivas y, por ende, constituyen espacios de ausencia simbólica que despoja a los sujetos de valor, lo que vuelve el cuerpo gordo, en uno negado.

Si bien, la gordura corporal genera un exceso de visibilidad en las personas, este "sobresalir" dentro del colectivo, se traduce en un sistemático proceso de carencia simbólica que le despoja del poder de significarse de otra manera, de asumirse como sujeto de derechos o bien, de ser reconocido por sus habilidades personales o profesionales. La gordura en el cuerpo reduce a las personas a los parámetros establecidos desde el prejuicio social por la grasa física.

A pesar de los múltiples intentos feministas por la reivindicación de los derechos de las personas no delgadas por existir desde su diversidad corporal. Lo cierto es que el sistema patriarcal de dominación ha asumido las estructuras educativas en nuestros países a tal punto, que no ha sido posible el establecimiento de espacios dentro de la academia donde se propicie la reflexión en torno a la gordofobia como un elemento presente dentro de las formas de vinculación entre los sujetos, asimismo, no ha sido posible repensar la gordura física lejos del paradigma médico, de modo que todo tipo de gordura se percibe como patológica, mientras la delgadez es asumida como símbolo de salud pocas veces cuestionada.

Por último, es necesario enfatizar en que son los aprendizajes asociados al cuerpo los que determinan la relación que los sujetos establecen con él. De ahí que exista una responsabilidad ineludible en los procesos educativos que se desarrollan en el ámbito privado, en las familias con niñas, niños y adolescentes, y aún más significativos dentro de los procesos educativos en el nivel institucional.

Sin embargo, reconocer la necesidad de trasformar la manera en la que educamos a los demás con relación a su cuerpo, conlleva un proceso reflexivo que inicia en el reconocimiento de la relación personal con nuestro ser, de modo que no es posible generar cambios hacia afuera, sin haber sanado las heridas derivadas de los aprendizajes negativos asociados al propio físico. 


\section{Referencias}

Álvarez, C. (2014). La cerda Punk. Ensayos desde un feminismo gordo, lesbiko, antikapitalista y antiespecista. Trio Editorial. https://n9.cl/hdd1

Chegne, N. (2012). Influencia de la escuela en la formación de los factores psicosocial de la desigualdad y los procesos de transformación social. Educación. (21) 41, 7-20. https://bit.ly/2NnxM4R

Darling, N. y Cumsille, P. (2003). Theory, measurement, and methods in the study of family influences on adolescent smoking. Addiction. (98) 1. 21-36. https://bit.ly/3oXXZEM

Díaz, M. y Díaz, M. (2008). Obesidad y autoestima. Enfermería Global, (7) 2. https://bit. ly/2XVXIXa

Magallares, A., Morales, J. y Rubio, M. (2010). Una aproximación a la obesidad desde el punto de vista psicosocial. Psicología general y aplicada. (63) 1-2. 59-71. https:// bit.ly/38WgzYg

Martín, M. (2002). La tiranía de la apariencia en la sociedad de las representaciones, en Revista Latina de Comunicación Social. 50. https://bit.ly/3qzSpJk

Martínez, A. (2004). La construcción social del cuerpo en las sociedades contemporáneas. Papers. 73. 127-152. https://bit.ly/3p3sglQ 
